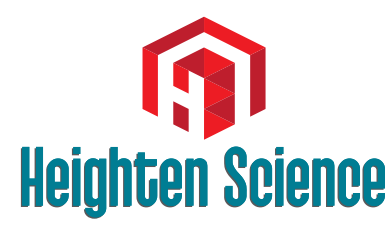

P U B L I C A T I O N S Corporation
*Address for Correspondence: Dr. Diaz-Lobato S, Pneumological Dipartment. Ramón y Cajal Universitary Hospital, Carretera de Colmenar Viejo, Km 9,100, 28034. Madrid. Spain, Tel: 003491336 8133; Email: sdiazlobato@gmail.com

Submitted: 27 February 2017

Approved: 29 May 2017

Published: 31 May 2017

Copyright: @ 2017 García G, et al. This is an open access article distributed under the Creative Commons Attribution License, which permits unrestricted use, distribution, and reproduction in any medium, provided the original work is properly cited.

\section{Avoiding confusion in high flow oxygen therapy concepts}

\author{
García G, Agosta M, Valencia P, Mercedes E, Sarhane Y and \\ Díaz-Lobato $S^{*}$ \\ Pneumological Department, Ramón y Cajal Universitary Hospital. Carretera de Colmenar Viejo \\ Km. 9.100, 28034, Madrid, Spain
}

Oxygen therapy is the main supportive treatment in hypoxemic respiratory failure and has traditionally been delivered using low and high flow devices. However, the maximal flow rates that these devices can deliver are limited because of the insufficient heat and humidity provided to the gas administered. Low flow devices such as the nasal cannula, conventional face mask and reservoir bag deliver a flow rate of up to $15 \mathrm{~L} / \mathrm{min}$ by administering more variable oxygen fractions (FiO2), depending on the patient's respiratory pattern, peak inspiratory flow and characteristics of the devices. Conventional high flow devices, such as venturi type masks, utilize a constant flow of oxygen through precisely sized ports, entraining the ambient air, using the Bernoulli principle, providing a more constant inspired oxygen fraction. However, they are less tolerated than nasal cannulas because they are less comfortable and the insufficient humidification and heating of the gas delivered [1].

In the last two decades, new devices have been developed to administer high humidified and heated flow through a nasal cannula (HFNC) that also allows the delivery of oxygen with a known FiO2 up to 100\%. In the literature, this technique has also been called mini CPAP (continuous positive airway pressure), transnasal insufflation, high nasal flow ventilation, high flow oxygen therapy, and high flow nasal cannula oxygen therapy [2].

It is considered that high flow nasal cannula has certain benefits compared to those of oxygen therapy previously detailed. HFNC manages a flow of more than $30 \mathrm{~L} / \mathrm{min}$, which is able to surpass the peak inspiratory flow of the patient, being able to reach values between $60-80 \mathrm{~L} / \mathrm{min}$ depending on the flow used. The gas source, which may be delivered by an air/oxygen blender, fans, or a flow generating turbine, is connected by an active humidifier to a nasal cannula and the FiO2 can be adjusted independently of the flow.

From a clinical point of view, there is some confusion between venturi and high flow nasal cannula devices. In the literature, both have been considered as high flow oxygen therapy devices. In our opinion this is not appropriate because the high nasal cannula flow is much more than a simple system for administering oxygen therapy [3]. Venturitype masks provide the patient with a gas mixture with a controlled FiO2, but do not exert additional benefits on the ventilator mechanics of the patient. Nevertheless, HFNC allows the delivery of a high flow, which can also add oxygen therapy, providing a series of physiological effects that imply an active treatment to respiratory failure.

Effects related to HFNC include the following:

1. Delivery of higher and more stable FiO2 values, because the flow delivered is greater than the patient's inspiratory demand. 
2. The anatomical dead space decreases by washing the nasopharynx, consequently increases alveolar ventilation. This improves the thoracoabdominal synchrony.

3. Respiratory work decreases because it acts as a mechanical stent in the airway and markedly attenuates inspiratory resistance.

4. The gas administered is warmed and humidified, improving mucociliar clearance, reducing the risk of atelectasis, improving ventilation perfusion and oxygenation ratio.

5. There is a CPAP-like effect. The dynamic positive espiratory airway pressure generated by HFNC reaches a value between $6-8 \mathrm{cmH} 20$ depending on the flow and the size of the cannula. This positive pressure distends the lungs and ensures their recruitment.

6. Pulmonary end-expiratory volume is higher with HFCN than with conventional high-flow oxygen therapy.

7. In addition, the technique is considered easy and simple for the medical staff and nurses, and can be used in different areas (emergency, hospitalization, critical care unit, weaning centers) and even at home [4].

Currently available evidence has demonstrated that HFNC therapy is an alternative for the treatment of acute hypoxemic respiratory failure, hypercapnic respiratory failure, acute heart failure, as rescue therapy preventive therapy in post-extubation respiratory failure and in specific conditions such as bronchoscopy [5].

We believe that high-flow nasal cannula treatment should not be confused with high flow oxygen therapy of venturi masks. According to detailed mechanisms of action, HFNC is not limited to being only an oxygen therapy system but also behaves as a true treatment that can be used in different clinical scenarios, generating physiological benefits that result in the reduction of respiratory work. In addition, in venturi type masks, the air is not humidified and complications such as dryness and nasal pain are common, generating a poor tolerance to oxygen therapy. The benefits of proper humidification and heating of the gas delivered with HFNC therapy allow better comfort and tolerance of the patient with easy adherence to the treatment. All this contributes to making HFNC be considered a technique of choice in patients with hypoxemic respiratory failure. The growth in its use associated with easy acceptance for patients and the expansion in its application show us that HFNC is a promising therapy.

\section{REFERENCES}

1. Spoletini G, Alotaibi M, Blasi F, Hill NS. Heated Humidified High Flow Nasal Oxygen in Adults, mechanism of action and clinical implications. Chest. 2015; 148: 253-261. Ref.: https://goo.gl/1NZOVm

2. Nishimura M. High Flow Nasal Cannula Oxygen Therapy in Adults. J Intensive Care. 2015; 3: 15. Ref.: https://goo.gl/YiBA4k

3. Díaz-Lobato S, Alonso JM, Carratalá JM, Mayoralas S. High Flow Nasal Oxygen is not an oxygen therapy device. Rev Prot Pneumol. 2015; 23: 51-52. Ref.: https://goo.gl/yTn1c7

4. Díaz-Lobato S, Mayoralas S. New approaches to the treatment of respiratory failure: High flow therapy. Med Clin (Barc). 2016; 147: 397-398. Ref.: https://goo.gl/irDqAD

5. Roca O, Hernández G, Díaz-Lobato S, Carratalá JM, Gutierrez R, et al. Current evidence for the effectiveness of heated and humidified high flow nasal cannula supportive therapy in adult patient with respiratory failure. Crit Care. 2016; 20: 109. Ref.: https://goo.gl/XAMA5H 